\title{
Unruptured aneurysm of the sinus of Valsalva presenting with isolated heart block: echocardiographic diagnosis and successful surgical
} repair

\author{
R A S AHMAD, S STURMAN, * R D S WATSON \\ From the Departments of Cardiology and ${ }^{\star}$ Medicine, Dudley Road Hospital, Birmingham
}

SUMMARY A 31 year old man presented with intermittent dizziness and electrocardiographic evidence of a severe conduction disturbance, with asystolic pauses of up to six seconds, and was treated by implantation of a permanent pacemaker. Echocardiography showed an enlarging aneurysm of the right sinus of Valsalva extending into the interventricular septum. This was confirmed by aortography, and successful surgical repair was carried out. The characteristic diastolic expansion of the aneurysm and the demonstration of continuity between the septal extension and the related aortic sinus were useful diagnostic features.

Aneurysmal dilatation and progressive enlargement of one of the sinuses of Valsalva, most often the right coronary sinus, normally goes undetected during life until rupture occurs. Rarely, the aneurysm presents with evidence of obstruction of the right ventricular outflow tract, aortic insufficiency, conduction abnormalities, or coronary artery compression in the absence of rupture. ${ }^{1}$ We report a patient who had a severe conduction disorder caused by extension of a congenital aneurysm into the interventricular septum and describe the echocardiographic features.

\section{Case report}

A 31 year old Pakistani man was admitted with a short history of intermittent vague dizziness associated with palpitation, near syncope, cold extremities, and mild dyspnoea. There was no history of recent chest pain or injury. On examination his pulse was normal with a regular rhythm at a rate of 70 beats/min, but with intermittent pronounced bradycardia. There were multiple, healed punched out ulcers on both shins. Examination of the fundi showed extensive bilateral retinal scarring with appearance of healed choroidoretinitis. Examination was otherwise unremarkable.

His electrocardiogram showed sinus rhythm, a

Requests for reprints to $\operatorname{Dr} R$ A S Ahmad, Department of Cardiology, Dudley Road Hospital, Dudley Road, Birmingham, B18 7QH. normal PR interval, right bundle branch block, and left anterior hemiblock. A chest radiograph was normal. Laboratory investigations showed normal blood count, erythrocyte sedimentation rate, serum electrolytes, and renal and liver function tests. Electrocardiographic monitoring showed symptomatic episodes of complete atrioventricular block with ventricular standstill lasting up to six seconds; a temporary transvenous pacemaker was inserted. Two days later a permanent VVI pacemaker was implanted, programmed to a rate of 50 beats $/ \mathrm{min}$.

Because he was young an echocardiographic examination was carried out, primarily to exclude heart muscle disease. This showed an echo free space high within the interventricular septum (fig 1) which appeared, on cross sectional examination, as a cystic mass near the left ventricular outflow tract. The appearances of the aortic and mitral valves, cardiac chambers, and aortic root were otherwise normal.

Further investigations were carried out because of the "cyst" in the ventricular septum seen on echocardiography and the cutaneous and retinal changes. There was no evidence of tuberculous infection. Blood cultures were sterile. Serological tests for syphilis, toxoplasma, and hydatid antibodies, and rheumatoid and antinuclear factors were negative. A Kveim skin test was negative.

One week later his conduction disturbance progressed to persistent complete atrioventricular block. Repeat echocardiography showed enlargement of the cyst in the septum and the characteristic 


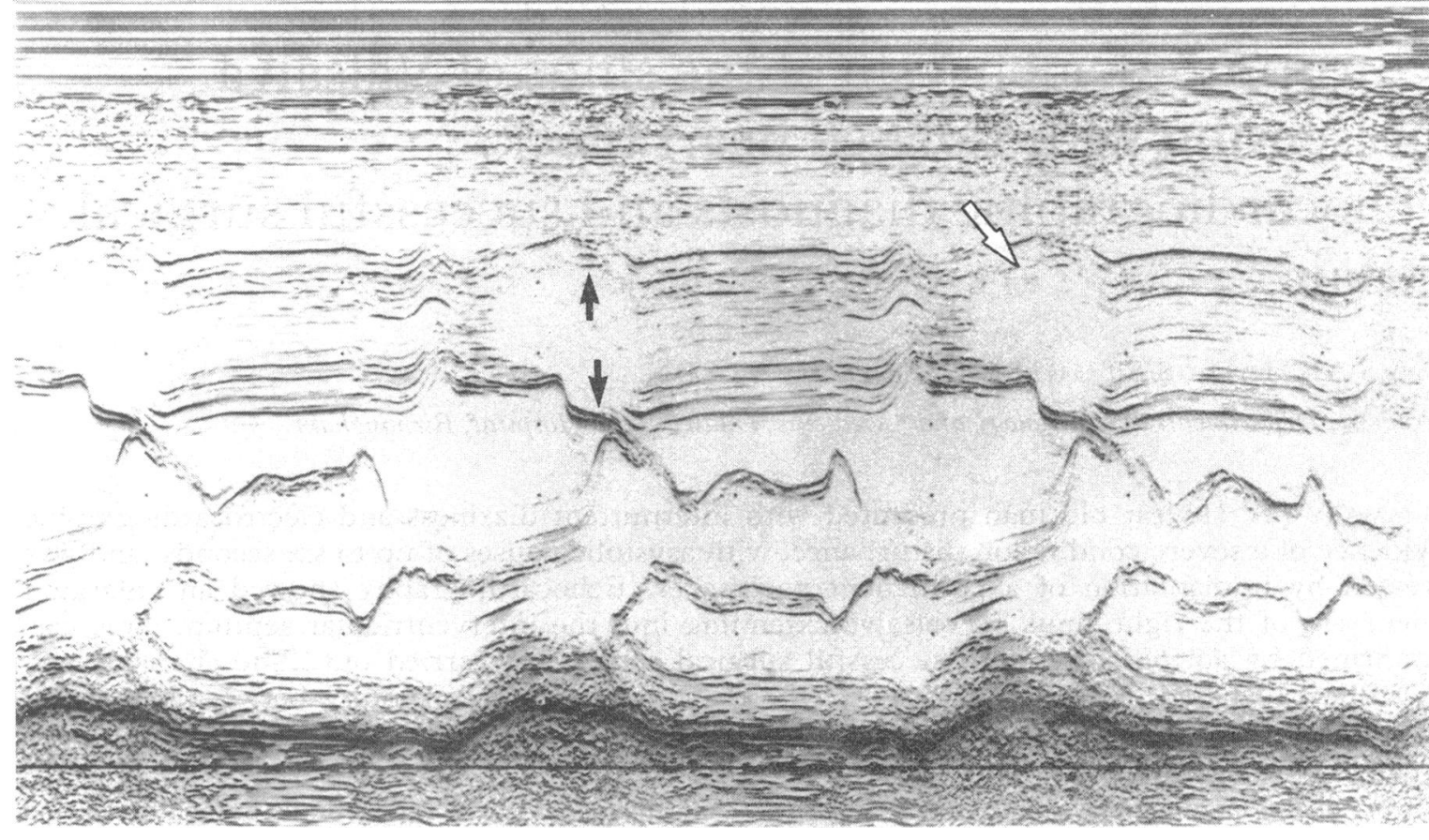

Fig $1 M$ mode echocardiogram showing the large echo free space (large arrow) within the grossly thickened interventricular septum. Expansion of the aneurysm during early diastole (small arrows) can be clearly seen, coinciding with maximum separation of the mitral valve leaflets.

appearances of an unruptured aneurysm of the sinus of Valsalva, as previously reported. ${ }^{23}$ Diastolic expansion and systolic "emptying" were apparent particularly in the long axis parasternal view. The echo free space extended rostrally, becoming continuous with the aortic root at the level of the right sinus of Valsalva.

The aneurysm did not appear to impinge appreciably on to the left or right ventricular outflow tracts. A root aortogram (fig 2) confirmed the presence of an unruptured aneurysm of the right coronary sinus extending into the interventricular septum. There was no evidence of aortic incompetence. Coronary angiography showed no abnormality.

In view of the progressive nature of the lesion we referred the patient for surgical repair five months after his initial presentation.

\section{OPERATIVE FINDINGS}

The heart was normal in size. Through the right ventricular free wall a mass could be felt in the upper part of the interventricular septum. The ascending aorta was normal and was opened under cardiopulmonary bypass. The entrance of the aneurysm, below the origin of the right coronary artery, measured approximately $1 \mathrm{~cm}$ in diameter. The right coronary cusp of the aortic valve was slightly distorted. The other two cusps and sinuses appeared normal. The aneurysm extended into the interventricular septum, bulging into the right ventricle and to a lesser extent $\vec{\phi}$ into the left ventricular outflow tract. The opening of $\stackrel{\varrho}{\beth}$ the aneurysm was closed with a Dacron patch and any distortion of the aortic valve was avoided. There was no intraoperative evidence of important aortic $\delta$ incompetence.

His subsequent course was uncomplicated. He 을 remained in complete atrioventricular block and was left with a ventricular demand pacemaker. At follow up three months later he was symptom free with only an insignificant early diastolic murmur. Repeat echocardiography showed disappearance of the echo $\tilde{O}$ free space within the interventricular septum. The N thickness of the septum had returned to normal.

\section{Discussion}

A congenital aneurysm of a single sinus of Valsalva is an uncommon anomaly attributed to deficiency or absence of media in the aortic wall behind the sinus. ${ }^{\prime} \stackrel{\mathbb{D}}{\circ}$ Enlargement of the aneurysm may occur and result in $\stackrel{\mathbb{Q}}{\mathbb{Q}}$ rupture, usually during the third or fourth decades of life. Over $90 \%$ of congenital aneurysms affect the 


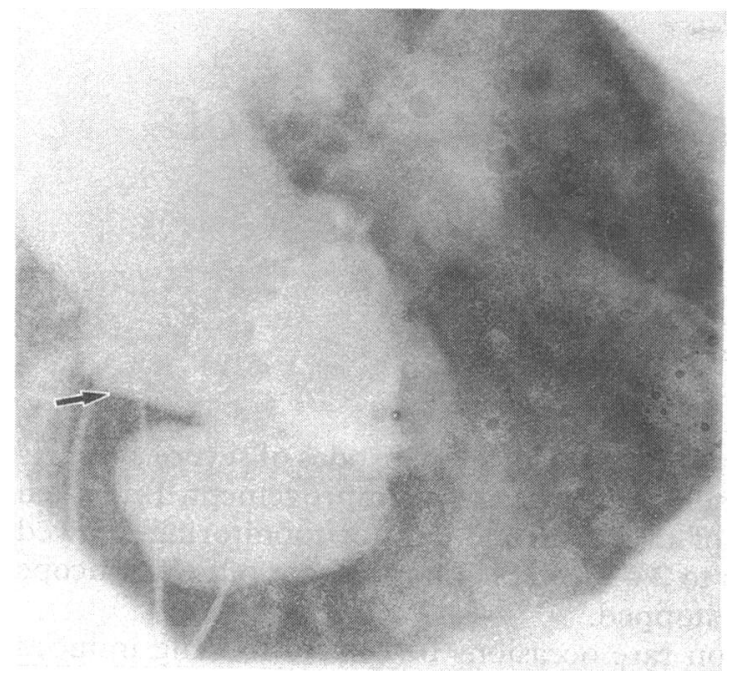

Fig 2 Root aortogram in left anterior oblique projection showing a normal left coronary sinus and the intact aneurysm of the right coronary sinus extending into the interventricular septum.

right or non-coronary sinuses and normally protrude, or rupture, into the right ventricle or right atrium. ${ }^{1}$ Rupture, most often in a young male patient, presents with chest pain, heart failure, or a loud continuous murmur. Extension of an aneurysm of a sinus of Valsalva into the interventricular septum to produce symptomatic conduction disturbance is very rare. Acquired aneurysms are mainly attributed to syphilis, infective endocarditis, or tuberculosis. In the absence of a clear cause a congenital aetiology is assumed.

The diagnosis has been made before rupture in patients presenting with aortic incompetence, bacterial endocarditis, or signs of obstruction of the right ventricular outflow tract with or without congestive cardiac failure. ${ }^{1-3}$

Echocardiography is useful in the diagnosis of ruptured aneurysms of the sinus of Valsalva ${ }^{4}$ and the diagnosis and follow up of unruptured aneurysms complicated by obstruction of the right ventricular outflow tract or infective endocarditis. ${ }^{25}$ Lewis and Agathangelou reported the echocardiographic diagnosis of an unruptured aneurysm of the right sinus of Valsalva in a patient with aortic regurgitation, infective endocarditis, and electrocardiographic evidence of bifascicular block. ${ }^{2}$ They commented on the value of demonstrating the continuity between the echo free space within the upper septum and the aortic root at the level of the right coronary sinus, which differentiates the condition from an aneurysm of the ventricular septum. Hands et al described the echocardiographic appearances of an unruptured aneurysm of the right coronary sinus with extension into the interventricular septum and the characteristic systolic "emptying" and diastolic expansion into the left ventricular outflow tract. ${ }^{3}$ This patient developed right bundle branch block and severe aortic incompetence requiring valve replacement.

In our patient the echocardiographic diagnosis was made by serendipity. There were no clinical features of aortic valve or aortic root abnormality and the echocardiogram was carried out to exclude heart muscle disease.

The course of unruptured congenital aneurysms is unknown. Survival for 19 years without operation was reported in a patient with a congenital aneurysm of the left sinus of Valsalva. ${ }^{7}$ There are no other reports of long term survival without operation. In our patient the rapid progression of the conduction disorder and increase in size of the septal extension prompted us to refer him for operation.

We thank Mr R Norton, consultant cardiothoracic surgeon, Walsgrave Hospital, Coventry, who operated on this patient and Dr G D Beevers, Reader in Medicine and honorary consultant physician, Dudley Road Hospital who shared his care.

\section{References}

1 Fishbein MC, Obma R, Roberts WC. Unruptured sinus of Valsalva aneurysm. Am J Cardiol 1975; 35:918-22.

2 Lewis BS, Agathangelou NE. Echocardiographic diagnosis of unruptured sinus of Valsalva aneurysm. Am Heart J 1984;107:1025-7.

3 Hands ME, Lloyd BL, Hung J. Cross-sectional echocardiographic diagnosis of unruptured right sinus of Valsalva aneurysm dissecting into the interventricular septum. Int J Cardiol 1985;9:380-3.

4 Engel PJ, Held JS, van der Bel-Kahn J, Spitz H. Echocardiographic diagnosis of congenital sinus of Valsalva aneurysm with dissection of the interventricular septum. Circulation 1981;63:705-11.

5 Faillace RT, Greenland P, Nanda NC. Rapid expansion of a saccular aneurysm on the left coronary sinus of Valsalva: a role for early surgical repair? $\mathrm{Br}$ Heart $\mathrm{J}$ 1985;54:442-4.

6 Kiefaber RW, Tabakin BS, Coffin LH, Gibson TC. Unruptured sinus of Valsalva aneurysm with right ventricular outflow obstruction diagnosed by twodimensional and Doppler echocardiography. $\mathrm{J} \mathrm{Am}$ Coll Cardiol 1986;7:438-42.

7 Martin LW, Hsu I, Schwartz H, Wasserman AG. Congenital aneurysm of the left sinus of Valsalva. Report of a patient with 19-year survival without surgery. Chest 1986;90:143-5. 